\title{
Highlighting the potential of peer-led workshops in training early-career researchers for conducting research with Indigenous communities
}

\author{
Gwyneth A. MacMillan ${ }^{a \star \dagger}$, Marianne Falardeau ${ }^{b \star \dagger}$, Catherine Girard ${ }^{c}$, Sophie Dufour-Beauséjour ${ }^{d}$, \\ Justine Lacombe-Bergeron $^{e}$, Allyson K. Menzies ${ }^{b}$, and Dominique A. Henrif \\ ${ }^{a}$ Department of Biological Sciences, Centre d'études nordiques, Université de Montréal, Montréal, QC \\ H2V 2S9, Canada; 'b Department of Natural Resource Sciences, McGill University, Macdonald Campus, \\ Ste. Anne de Bellevue, QC H9X 3V9, Canada; 'Centre d'études nordiques, Université Laval, Québec, \\ QC G1V 0A6, Canada; ${ }^{\mathrm{d}}$ Centre d'études nordiques, Centre Eau Terre Environnement, Institut national \\ de la recherche scientifique, Québec, QC G1K 9A9, Canada; ${ }^{\mathrm{T}}$ Mine of Knowledge, Department \\ of Biological Sciences, Université de Montréal, Montréal, QC H2V 2S9, Canada; ${ }^{\mathrm{f}}$ Environment and \\ Climate Change Canada, Montréal, QC H2Y 2E7, Canada \\ *gwyneth.anne.macmillan@umontreal.ca; marianne.falardeau-cote@mail.mcgill.ca \\ ${ }^{\dagger}$ Co-first authorship
}

\section{OPEN ACCESS}

\section{Citation: MacMillan GA, Falardeau M, Girard C, Dufour-Beauséjour S, Lacombe- Bergeron J, Menzies AK, and Henri DA. 2019. Highlighting the potential of peer-led workshops in training early-career researchers for conducting research with Indigenous communities. FACETS 4: 275-292. doi: I 0. I I 39/facets-20 I 8-0046 \\ Handling Editor: Christopher Scott Findlay}

Received: November 6, 2018

Accepted: March 29, 2019

Published: July 8, 2019

Copyright: (c) 2019 MacMillan et al. This work is licensed under a Creative Commons Attribution 4.0 International License (CC BY 4.0), which permits unrestricted use, distribution, and reproduction in any medium, provided the original author(s) and source are credited.

Published by: Canadian Science Publishing

\section{Abstract}

For decades, Indigenous voices have called for more collaborative and inclusive research practices. Interest in community-collaborative research is consequently growing among university-based researchers in Canada. However, many researchers receive little formal training on how to collaboratively conduct research with Indigenous communities. This is particularly problematic for earlycareer researchers (ECRs) whose fieldwork often involves interacting with communities. To address this lack of training, two peer-led workshops for Canadian ECRs were organized in 2016 and 2017 with the following objectives: (i) to cultivate awareness about Indigenous cultures, histories, and languages; (ii) to promote sharing of Indigenous and non-Indigenous ways of knowing; and (iii) to foster approaches and explore tools for conducting community-collaborative research. Here we present these peer-led Intercultural Indigenous Workshops and discuss workshop outcomes according to five themes: scope and interdisciplinarity, Indigenous representation, workshop environment, skillful moderation, and workshop outcomes. Although workshops cannot replace the invaluable experience gained through working directly with Indigenous communities, we show that peer-led workshops can be an effective way for ECRs to develop key skills for conducting meaningful collaborative research. Peer-led workshops are therefore an important but insufficient step toward more inclusive research paradigms in Canada.

Key words: early-career researchers, collaborative research, Indigenous communities, peer-led training, workshop evaluation, cultural awareness

\section{Introduction}

For decades, Indigenous voices have been calling for changes to research approaches in Canada that exclude, marginalize, or appropriate Indigenous knowledge. Many Indigenous communities have 
been asking for a greater say in decisions about research priorities and ethical methodologies as well as data storage, interpretation, and sharing (Hall 2005; NRI and ITK 2007; Wilson 2008; Kovach 2009; GC 2014; INQ 2017; ITK 2018). In this context, awareness of the importance of collaborative approaches to research with Indigenous communities is increasing within many Canadian research institutions (Castleden et al. 2012; Adams et al. 2014; Johnson et al. 2015) resulting in a movement away from conducting research on or in Indigenous communities to conducting research with communities (Tobias et al. 2013; Vogel 2015). A fundamental step toward integrating collaborative approaches into mainstream research is training university-based researchers to work with Indigenous communities in Canada.

Community-collaborative research can be defined as an overarching term that includes a continuum of different research approaches, which "involves engaging local communities and individuals in the research process with the goal of sharing or co-generating knowledge to understand complex problems" (Tondu et al. 2014). This continuum of approaches includes science communication and outreach, community consultation, community-based monitoring, community-engaged research, and participatory research with co-production of knowledge. The nature and extent of appropriate community participation can vary depending on several factors, including the research questions and goals, as "not all types of northern research will require, or inspire, the same level of community involvement" (NRI and ITK 2007, p. 10). Collaborative methodologies remain under-represented in some natural science disciplines (Brunet et al. 2014a; Johnson et al. 2015), and some projects may offer fewer opportunities for local involvement such as studies conducted far from communities using remote data loggers or focusing on noncharismatic species (NRI and ITK 2007; Gagnon and Berteaux 2009).

Collaborative research with meaningful community engagement can have positive outcomes for both researchers and Indigenous community members, including improved quality and legitimacy of research, capacity and trust building, and respectful integration of local knowledge (Jack et al. 2010; Ford et al. 2013; Tobias et al. 2013; Brunet et al. 2014a; Mantyka-Pringle et al. 2017). However, developing and implementing long-term and meaningful engagement can be difficult. Challenges experienced by researchers include limited funding and time, academic constraints, and insufficient professional recognition for the efforts involved in collaborating (Koster et al. 2012; Tobias et al. 2013; Adams et al. 2014; Brunet et al. 2014b; Ayre et al. 2018). Differences in worldviews between Indigenous community members and non-Indigenous researchers can also yield divergent approaches to, and expectations of, collaborative research. Negative outcomes may arise during collaborative research, such as the perpetuation of power imbalances, breaches of community customs, and miscommunication about ethical guidelines (Mitchell and Baker 2005; Koster et al. 2012; Ayre et al. 2018). Skills in cross-cultural engagement are therefore essential for conducting collaborative research with positive outcomes (Adams et al. 2014; Johnson et al. 2015).

Early-career researchers (ECRs) face additional challenges than those faced by more established researchers. ECRs need to be productive and publish within the time span of a graduate degree (2-5 years) and may be required to do so with smaller research budgets and more personal financial insecurity (Tondu et al. 2014). When resources, support, and incentives are lacking, ECRs may engage with Indigenous communities chiefly as a result of personal ethics and values (Brunet et al. 2014b). While useful ethical guidelines for conducting community-collaborative work do exist (NRI and ITK 2007; INQ 2017; ITK 2018), ECRs may be limited in their ability to apply these recommendations as they typically conduct their research as part of pre-established projects. For instance, OCAP ${ }^{\mathrm{TM}}$ (Ownership, Control, Access, and Possession) is a broadly recognized standard for issues of access and ownership of research data with First Nations in Canada (First Nations Information Governance Centre 2014). However, ECRs cannot always address issues around data 
ownership when they are working as part of pre-established projects where they have limited control over study design and data sharing. Many ECRs in the natural sciences are also not aware of these existing ethical guidelines, as training on conducting community-collaborative research with Indigenous communities is rarely included in natural science curricula (Bousquet 2012; Tondu et al. 2014).

Without formal training, many ECRs are self-taught and must learn through trial and error when carrying out collaborative research with Indigenous communities. In a 2014 survey of Arctic ECRs from 22 countries, the majority of respondents reported wanting to involve more Indigenous peoples in their research, yet only half felt that they had the basic knowledge to do so. In the same survey, natural scientists rated their knowledge on conducting research with northern Indigenous communities much lower than social scientists (Sjöberg et al. 2019). Opportunities do currently exist for Canadian researchers (including ECRs) who want to develop skills for working with Indigenous communities, including massive online courses and courses on research ethics, such as the Carleton University Institute on the Ethics of Research with Indigenous peoples. However, formal training for students is rare and courses offered outside universities can be prohibitively expensive for ECRs. As Indigenous communities call for a greater say in research, it is essential to improve training that provides tools and approaches for working collaboratively with Indigenous partners to the future generation of researchers (Tondu et al. 2014).

Peer-led workshops can be part of the solution, as they are an effective training technique that enhances interactivity, engagement, motivation, and student learning (Preszler 2009; Deakin et al. 2012; Ouellet Dallaire et al. 2018). Here, we present two peer-led workshops entitled "Intercultural Indigenous Workshops" that were held in 2016 and 2017 in Montréal, Québec, Canada, with the aim of preparing ECRs to work together with Indigenous communities. Specific workshop objectives were to: (i) cultivate awareness about Indigenous cultures, histories, and languages; (ii) promote sharing of Indigenous and non-Indigenous ways of knowing; and (iii) foster approaches and explore tools for conducting community-collaborative research. In this perspective, we discuss the design, implementation, and outcomes of our peer-led workshops in relation to the three objectives described above. We also explore challenges and ways of improving this type of training for ECRs conducting collaborative research with Indigenous communities.

\section{Positionality of authors and definitions}

The authors of this paper all identify as ECRs. ECRs are defined here as undergraduate and graduate students, post-doctoral fellows, and new principal investigators. The authors include six workshop organizers and a government-based researcher who participated in the 2016 workshop as an invited speaker and provided mentorship to the organizers (D.A. Henri). All authors identify as female researchers; two are Métis and the others are non-Indigenous. We acknowledge that our own perspectives and worldviews will have influenced the interpretations presented here.

In referring to researchers working with Indigenous communities in this article we include researchers who work directly with Indigenous partners as well as those whose projects take place in (or near) Indigenous communities. This article seeks to address issues in training for ECRs whose projects are situated all along a continuum of community involvement in research, from science outreach to participatory research. Indigenous community is used here to describe a group of people with a shared cultural identity, traditions, and ways of life, which has the capacity to act or express itself as a collective (GC 2014). Indigenous communities can be territorial, organizational, or a community of interest (ACUNS 1982). 


\section{The Intercultural Indigenous Workshops}

In this section, we describe the two Intercultural Indigenous Workshops held in 2016 and 2017. These two workshops were organized by volunteer committees composed of five to six ECRs in the natural sciences (representing three universities in Québec, Canada) and one university employee. The committees organized the workshops because of a perceived lack of training for both themselves and their peers on conducting community-collaborative research. The workshops were focused on ECRs because they are typically more involved in fieldwork and in the day-to-day interactions with Indigenous communities than senior researchers. Faculty members aided in securing funding but were not directly involved in workshop organization. Both events were publicized using departmental and research-group mailing lists, posters at universities, word of mouth, and social media. Funding was obtained from grants and participant registration fees. This section will provide a brief description of the workshops and quantitative information on workshop participation. More details on workshop schedules and budgets, round-table discussion structure and questions, a summary of the 2016 participatory research interactive activity, and post-workshop survey questions are provided in Supplementary Material 1.

In April 2016, a 1-d workshop entitled “Atelier autochtone interculturel: Savoirs scientifiques et locaux en recherche nordique" ("Intercultural Indigenous Workshop: scientific and local knowledge in northern research") was held in French at the Université de Montréal. The morning consisted of hour-long presentations by invited facilitators who discussed: (i) how to foster collaboration between researchers and Indigenous community members in Québec, (ii) an Inuit perspective on the history of researchers working in the North, and (iii) Indigenous cultures and languages in Canada. The afternoon consisted of a round-table discussion on how to encourage cooperation, knowledge transfer, and the sharing of perspectives between scientific and northern Indigenous communities. Roundtable participants included two university professors, two university students, and one postdoctoral fellow; one of whom identified as Indigenous. After the round-table discussion, an interactive activity consisting of small group discussions on participatory research was facilitated by a government researcher. This session led to the creation of a document summarizing the group discussions and providing resources for ECRs (see Supplementary Material 1). The workshop ended with an evening networking event featuring traditional food tasting and a performance by Inuit throat singers.

In November 2017, a 2-d workshop entitled "Intercultural Indigenous Workshop: Sharing perspectives and experiences of research in Canada's North" was held at McGill University. The first day consisted of a full-day workshop on building cultural awareness of Indigenous cultures and was led by an Indigenous facilitator. Canada's historic relationships with First Nations, Inuit, and Métis were explored during this first day. In the evening, a public event was organized that included two keynote presentations and a round-table discussion. The keynote facilitators, one of whom identified as Indigenous, spoke about merging traditional and scientific ecological knowledge and about the importance of youth education in Indigenous communities. The theme for the round-table discussion was "Sharing perspectives and experiences of research in Canada's North." Round-table participants included a university professor, a postdoctoral fellow, a special advisor on Aboriginal initiatives, an Indigenous artist, the co-founder of an Indigenous organization, and a doctoral student; three of these participants identified as Indigenous. The round-table discussion was followed by a networking event that included food from a local Indigenous caterer, an Inuit throat singing performance, and jewellery sold by Indigenous artisans. The second day of the workshop was an interactive, arts-based, experiential workshop focused more specifically on the history of First Nations and led by an Indigenous facilitator. The goal of the experiential workshop was to explore First Nations' historical, political, and social issues by re-enacting historical scenarios and by using theatrical techniques. An Indigenous Elder was present to offer guidance. 
Table 1. Affiliation of workshop participants by year.

Participant affiliation

University/academic

Government of Canada

Other organizations

Number of academic institutions

Number of academic departments $2016(n=32)$

$2017(n=48)$

\begin{tabular}{lc}
32 & 46 \\
- & 2 \\
- & 1 \\
9 & 12 \\
8 & 24 \\
\hline
\end{tabular}

Note: Individual participants can be counted in multiple categories.

Table 2. Workshop participants' fields of study, degree level, and gender by year.

\begin{tabular}{|c|c|c|c|c|c|c|c|c|c|c|c|}
\hline Total participants & $n$ & $\%$ & Field of study & $n$ & $\%$ & Degree & $n$ & $\%$ & Gender & $n$ & $\%$ \\
\hline \multicolumn{12}{|l|}{2016} \\
\hline \multirow[t]{5}{*}{ Day 1: actual/registered } & $32 / 43$ & 74 & Natural sciences & 24 & 75 & Bachelor's student & 7 & 22 & Female & 21 & 65 \\
\hline & & & Social sciences & 5 & 15 & Master's student & 10 & 31 & Male & 11 & 35 \\
\hline & & & Health sciences & 3 & 10 & Ph.D. student & 12 & 38 & & & \\
\hline & & & & & & Post-doc & 3 & 9 & & & \\
\hline & & & & & & Other & 0 & - & & & \\
\hline \multicolumn{12}{|l|}{2017} \\
\hline Day 1: actual/registered & $48 / 59$ & 81 & Natural sciences & 23 & 48 & Bachelor's student & 4 & 8 & Female & 37 & 77 \\
\hline Day 2: actual/registered & $15 / 30$ & 50 & Social sciences & 14 & 29 & Master's student & 19 & 40 & Male & 11 & 23 \\
\hline Webinar & 23 & - & Health sciences & 10 & 21 & Ph.D. student & 15 & 31 & & & \\
\hline \multirow[t]{2}{*}{ Networking } & 92 & - & Other & 1 & 2 & Post-doc & 9 & 19 & & & \\
\hline & & & & & & Other & 1 & 2 & & & \\
\hline
\end{tabular}

The 2016 workshop brought together 32 participants from eight fields of research who were affiliated with nine different universities in the province of Québec, Canada (Table 1). The majority of participants were female graduate students (>60\%) in the natural sciences (Table 2). In 2017, the first full-day workshop involved 48 participants from 24 disciplines who were affiliated with 10 Canadian universities, as well as representatives from the federal government and an Indigenous organization (Tables 1 and 2). Overall, the participants came from a greater diversity of research fields and institutions in 2017. The 2017 public evening event attracted 92 people, with an additional 23 people registered via webinar. The experiential workshop in 2017 had 15 participants (14 female) who were also mostly in the natural sciences. Similar to 2016, the dominant group of participants in 2017 were female graduate students $(>70 \%)$ in the natural sciences (Table 2). Fewer participants stated that they had previous experience working in northern research in 2017 compared to 2016 ( $33 \%$ had worked in northern research in 2017 vs. $53 \%$ in 2016). In a recent study, female ECRs rated their knowledge of Indigenous peoples more highly and found more benefits in involving Indigenous peoples in their research than their male counterparts (Sjöberg et al. 2019). These trends may have contributed to the predominance of female natural scientists at our workshops. 


\section{Workshop assessment methods}

The organizing committees collected post-workshop feedback through a variety of methods from participants, facilitators, and organizers. Feedback focused on the workshop design, implementation, and overall impressions. Quantitative data were compiled from attendance records for participants on the following topics: (i) affiliation to university/department or other organization, (ii) role within university/department or other organization (e.g., student, faculty, employee), and (iii) gender. Gender was assigned based on first names and verified with gender name lists by country (Larivière et al. 2013). Members of the organizing committees $(n=13)$ provided oral and (or) written feedback (via email) on their overall impressions of the workshops. Facilitators were sent questions via email $(n=8)$ or asked questions via semi-structured, oral interviews that were transcribed afterwards $(n=1)$ with informed consent (see Supplementary Material 1 for interview questions).

After the workshops, all participants received email invitations to anonymously complete a workshop evaluation survey, which included 9-10 multiple-choice and short-answer questions. The overall aim was to evaluate the strengths and weaknesses of the workshops (see Supplementary Material 1 for participant survey questions). We received 19 responses in 2016 and 18 in 2017. Categorical and open-ended questions covered participant opinions on various aspects of the workshops, including: schedule, workshop themes, presentations, and networking events. Categorical questions included two different scales to measure appreciation: (i) "Very Poor", "Poor", “Average", "Good”, or "Very Good" and (ii) "Not Useful”, "Somewhat Useful”, "Useful”, or "Very Useful." In total, 100\% of organizers, $53 \%$ of facilitators and $46 \%$ of participants (who both registered and attended a workshop) provided feedback.

We used mixed methods to describe and discuss perspectives from participants, facilitators, and organizers on their workshop experiences. We adapted a framework from Cervero (1984) to use four workshop evaluation categories: (i) workshop design and implementation, (ii) learner participation, (iii) learner satisfaction, and (iv) workshop outcomes (Fig. 1). Learner participation was evaluated using descriptive statistics and qualitative description (Sandelowski 2000); all other categories were explored with qualitative description. Two authors independently coded the qualitative information available (interview transcripts, surveys, emails, debriefing notes) based on each of the evaluation categories from the Cervero (1984) framework. Through thematic analysis, the categories were subsequently organized into fundamental and interrelated themes that best reflected the main ideas raised by the participants, facilitators, and organizers. This paper explores five themes associated with workshop format and outcomes: (i) scope and interdisciplinarity, (ii) Indigenous representation, (iii) workshop environment, (iv) skillful moderation, and ( $v$ ) workshop outcomes (Fig. 1). The discussion reflects on how each of these themes contributed to workshop success, which was evaluated based on the three workshop objectives.

\section{Ethics approval and informed consent}

For this type of workshop discussion and evaluation, ethics approval was not required. The authors consulted with two university research ethics boards at McGill University and the Université de Montréal (G.A. MacMillan and M. Falardeau, personal communication, 2018). Both research ethics boards concluded that, as per Section 2.5 of the Tri-Council Policy Statement: Ethical Conduct for Research Involving Humans (Canadian Institutes of Health Research, Natural Sciences and Engineering Research Council of Canada, and Social Sciences and Humanities Research Council of Canada 2014, p. 18), "Program evaluation activities, and performance reviews [... ] do not constitute research for the purposes of this Policy, and do not fall within the scope of REB [research ethics board] review." We therefore incorporated anonymous participant feedback into this article as the purpose of this perspective was to evaluate workshop success. We did not obtain formal consent from 


\section{Qualitative Data}

Written feedback by organizers

Email exchanges by organizers

Participant emails

Participant surveys

Facilitator feedback

\section{Quantitative Data}

\section{Registration data}

Participant surveys

\section{Workshop Evaluation Categories}

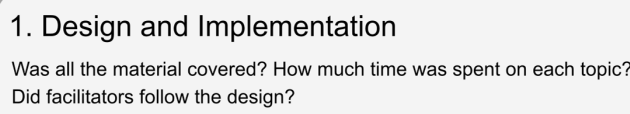

1. Design and Implementation

Was all the material covered? How much time was spent on each topic? Did facilitators follow the design?

\section{Learner Participation}

Who were the participants? Were these the intended participants? How involved were the participants?

\section{Learner Satisfaction}

How was the workshop judged by learners?

Did topic sequence promote effective learning?

Was the setting conducive to learning?

\section{Outcomes}

Did the participants learn new knowledge, skills or attitudes?

Did participants apply learning after the workshop?

What workshop characteristics were associated with outcomes?

\section{Discussion Themes}

Scope and Interdisciplinarity

Indigenous Representation

Workshop Environment

Skillfull Moderation

Workshop Outcomes

Fig. 1. Diagram of the workshop evaluation method using quantitative data, qualitative description, and workshop evaluation categories from the framework in Cervero (1984). The diagram shows the five fundamental and interrelated discussion themes that reflect key ideas raised by workshop participants, facilitators, and organizers.

workshop participants as we could not retroactively seek their consent from anonymous survey responses. Furthermore, we sought informed consent from all facilitators before incorporating their feedback. Participating facilitators were informed that the goal of the semi-structured interviews was to collect feedback about their workshop experience to be used in this manuscript. All interviewed facilitators gave written or oral consent for their feedback (and quotes) to be used in this paper (see Supplementary Material 1: Semi-Structured Interview Questions for Facilitators, Question 7).

\section{Discussion}

I never had the chance to attend a training day like this and I wish that I could have, as it would have helped me a lot. I am very happy to see that there are now resources like this being developed for the new generation of researchers ... this means that times are changing.

Workshop facilitator in 2016

For the most part, the workshops were effectively implemented and well-received by participants and facilitators. There was an overall high degree of satisfaction; the majority of survey participants rated their overall workshop appreciation as either "Good" or "Very Good" (100\% in 2016, 90\% in 2017). Most survey participants also reported that the workshops were either "Very Useful" or "Useful" to their research (72\% in 2016 and 67\% in 2017). Adjectives frequently used to describe the workshop by participants included "excellent", "well-organized”, "interesting”, "pertinent”, and "useful." All invited facilitators who provided feedback shared their appreciation for the workshops and agreed to participate in future editions. One non-Indigenous facilitator in 2016 acknowledged the historical lack of training on these subjects and highlighted the current need for such workshops (see above quote). While general appreciation was high for both workshops, participant satisfaction for some activities was mixed. In this discussion, we describe the five themes that emerged from the analysis and evaluate the success of our workshop by relating these themes back to our three workshop objectives. These objectives were to support ECR training for conducting research with Indigenous communities by: $(i)$ cultivating awareness of Indigenous cultures, histories, and languages; 
(ii) promoting sharing of Indigenous and non-Indigenous ways of knowing; and (iii) fostering approaches and explore tools for conducting collaborative research with Indigenous communities.

\section{Scope and interdisciplinarity}

The workshop showed me how social sciences play an important role in the field, and also how social sciences are complementary with "pure" sciences to determine, with local communities, how to use a collaborative approach and to decolonize relationships.

Workshop participant in 2016

One of the major challenges of organizing workshops that addressed complex issues around researcher and Indigenous collaborations was determining the scope of the workshops. The organizing committees put a great deal of thought into this. Should the workshops focus on a specific scientific field (e.g., natural sciences, social sciences, or both)? Should they be targeted to northern researchers or to all researchers? Should workshops emphasize relationship building with one Indigenous group or with Indigenous peoples in Canada more broadly? Our thematic analysis revealed that our effectiveness in meeting the workshop objectives depended largely on decisions made about scope and interdisciplinarity.

Both narrowly and broadly targeted workshops proved successful in cultivating cultural awareness of Indigenous cultures, histories, and languages. The 2016 edition had a narrower scope, focusing on northern Indigenous communities, in particular Inuit, whereas the 2017 edition focused on First Nations, Inuit, and Métis. Survey participants in 2016 commented that learning facts about Inuit culture and ways of life was a highlight of the workshop. In 2017, one participant stated that the workshop was "eye-opening" and an important reminder of the context and history of Indigenous peoples in Canada. While some participants felt that the 2017 workshop was too broad and not targeted to researchers, a participant mentioned that researchers would still keep in mind the workshop messages when working with Indigenous groups, despite the broad scope.

The target audience shifted from the first to the second workshop-from a narrower to a broader audience-and both formats helped to promote interdisciplinary discussions and the sharing of diverse perspectives. Based on participant feedback, the organizers broadened the target audience of the second workshop (2017) to include the social and health sciences, as well as to participants beyond academia. Indeed, having a great diversity of disciplines among workshop participants, facilitators, and organizers, can help foster interdisciplinary thinking, openness, and collaboration (Bridle et al. 2013). The sharing of perspectives was also encouraged at both workshops by incorporating time for discussion and reflection at social events. Despite more homogeneous participant profiles in 2016 (75\% in the natural sciences), workshop facilitators were from diverse backgrounds which allowed for interdisciplinary encounters. One 2016 facilitator stated: "My general impression was that students in the 'hard' sciences were very receptive to knowledge from the social sciences. They wanted to know more." For both workshops, stimulating interdisciplinary discussions was key to sharing ways of knowing across Indigenous and non-Indigenous knowledge systems.

Although both workshops were effective at achieving the first two objectives of the workshops, the third objective of fostering approaches and exploring specific tools for conducting collaborative research was better served by the narrower workshop scope in 2016. Participants appreciated content that was clearly related to their own research practice; they were less satisfied in 2017 when the applicability of workshop content to their research was less clear. A narrower scope in 2016 also enabled participants to build interest around a common theme, which enabled discussions on field-specific methodologies for conducting collaborative research (Bridle et al. 2013). Overall, our analysis showed 
that a successful formula for meeting the workshop objectives was to (i) have a specific target audience, (ii) ensure a diversity of facilitators to engage with workshop participants, and (iii) focus on relationship building with one or a few Indigenous groups. We therefore recommend organizing multiple, specialized workshops tailored to specific groups to promote community-collaborative research approaches, while also building cultural awareness and sharing perspectives.

\section{Indigenous representation}

Simply that this event was organized was a high point for me. Indigenous peoples are often forgotten in research and this type of event can help build better relationships.

Workshop facilitator in 2017

An essential aspect of the peer-led workshops was the inclusion of a diversity of cultural identities and experiences. Indigenous facilitation was indispensable for achieving key workshop objectives, especially raising awareness about Indigenous cultures, histories, and languages and promoting Indigenous ways of knowing. Significant effort went into having diverse facilitators from Inuit, Métis, Lakota, and Mohawk nations/communities. Survey participants often reported that their preferred workshop activities were those led by Indigenous facilitators and some participants requested more Indigenous representation, including suggestions for an all-Indigenous speaker program.

However, integrating a diversity of Indigenous facilitators posed some challenges for the ECR-led organizing committees who had limited professional networks. It was challenging to find Indigenous speakers, many of whom were highly sought after for this type of training. Furthermore, soliciting help from nonacademic facilitators was difficult, as participating without compensation in workshops is rarely part of nonacademic professional responsibilities. Feedback from some facilitators suggested addressing this challenge by increasing Indigenous participation on the organizing committees and partnering with Indigenous organizations to plan future events. Ensuring enough funding to be able to provide compensation for a diversity of members on the organizing committees, and for nonacademic guests, would help address these challenges.

In addition, the organizing committees did not have sufficient funding to pay for facilitators to fly from Indigenous communities, restricting recruitment to facilitators who lived near Montréal, Québec (Canada). Future workshops could use virtual communication technology (e.g., Skype) to connect Indigenous facilitators from remote locations-such as Canada's northern territories-with participants. Virtual communication can be an effective way to connect learners across geographical barriers (Mercier and Leonard 2000). However, virtual discussions may be less effective than in-person meetings for relationship building. Overall, the challenges faced by the organizing committees in their efforts to have a high Indigenous representation were typical of ECR-led workshops. More extensive professional networks and greater access to funding would likely have enhanced the workshops' success in meeting the objectives.

Nonetheless, effectively sharing perspectives at the workshops was fostered by diverse Indigenous and non-Indigenous facilitators who were able to draw on different experiences and contextualize the world of collaborative research for ECRs. Indigenizing training (and research) indeed requires close collaboration between researchers and Indigenous peoples (Kitchen and Raynor 2013). A diversity of Indigenous facilitators cultivated cultural awareness among participants and exposed them to different ways of knowing. Yet, many of the Indigenous facilitators had not conducted research (although some had been research participants) and the non-Indigenous researchers were able to provide insights into the specifics of collaborative research approaches. Ultimately, key 
workshop objectives were achieved thanks to significant efforts made to include strong Indigenous representation at the workshops, and to ensure a balance between Indigenous and non-Indigenous facilitators.

\section{Workshop environment}

Perhaps consider booking out space in the Kahnawake Mohawk Territory to do the workshop and presentation there. Context matters!

Workshop facilitator in 2017

Our analysis found important connections between the physical environments of the workshops and the participants' experience, especially in an intercultural context. Both workshops were held on university grounds and in settings that were typical of academic conferences (Fig. 2). This environment is likely comfortable for university students and faculty members, but may be less so for nonacademics and Indigenous participants. On the one hand, hosting these peer-led workshops at the university encouraged student participation and meant that the venues were easy to reserve for student committees. On the other hand, many of the Indigenous facilitators emphasized that the physical space was not conducive to intercultural learning. For instance, it was not possible to host a smudging ceremony (which involves burning medicinal herbs) at one workshop because of university building regulations. One Indigenous facilitator also commented on the importance of geographical location (see above quote). Some facilitators suggested that the room configurationfor instance, a rectangular table on an elevated stage for the inaccurately named "round-table" discussion-did not promote cultural awareness and the sharing of perspectives. Although territorial acknowledgements were conducted at the beginning of each workshop, the overall academic context and room configurations may have created a disconnect between the workshop experience and its objectives. Opting for a venue located in an Indigenous community, setting-up rooms in a culturally appropriate way, and incorporating more Indigenous teaching methods such as storytelling would have been a more effective way of sharing Indigenous ways of knowing (Kovach 2009; Castleden et al. 2013).

The importance of the workshop environments to achieving workshop objectives also extended beyond the physical space. The use of diverse learning techniques, the inclusion of socio-cultural activities and the type of food served influenced the success of the workshops. At the Intercultural Indigenous Workshops, activities ranged from lectures to round-table discussions to interactive activities involving talking circles and role-play. Participants frequently reported that the variety of activities was a highlight. The Indigenous catering and socio-cultural activities (throat singing, Indigenous artisans) were also highly appreciated and were seen as opportunities to build awareness about Indigenous cultures. One organizer reported that: "We managed to create space for exchanges between students, researchers, and Indigenous peoples through different formats [...], but we also broke down academic barriers by offering opportunities for informal exchanges through having [Indigenous] artists sell their crafts and food sharing." Overall, our analysis found that experiential learning and socio-cultural activities significantly contributed to raising cultural awareness among workshop participants (Castleden et al. 2013).

\section{Skillful moderation}

Leading this kind of activity is quite challenging to pull off in my eyes, because the issues raised reach deep into the psychological realm and personal experiences of participants. 


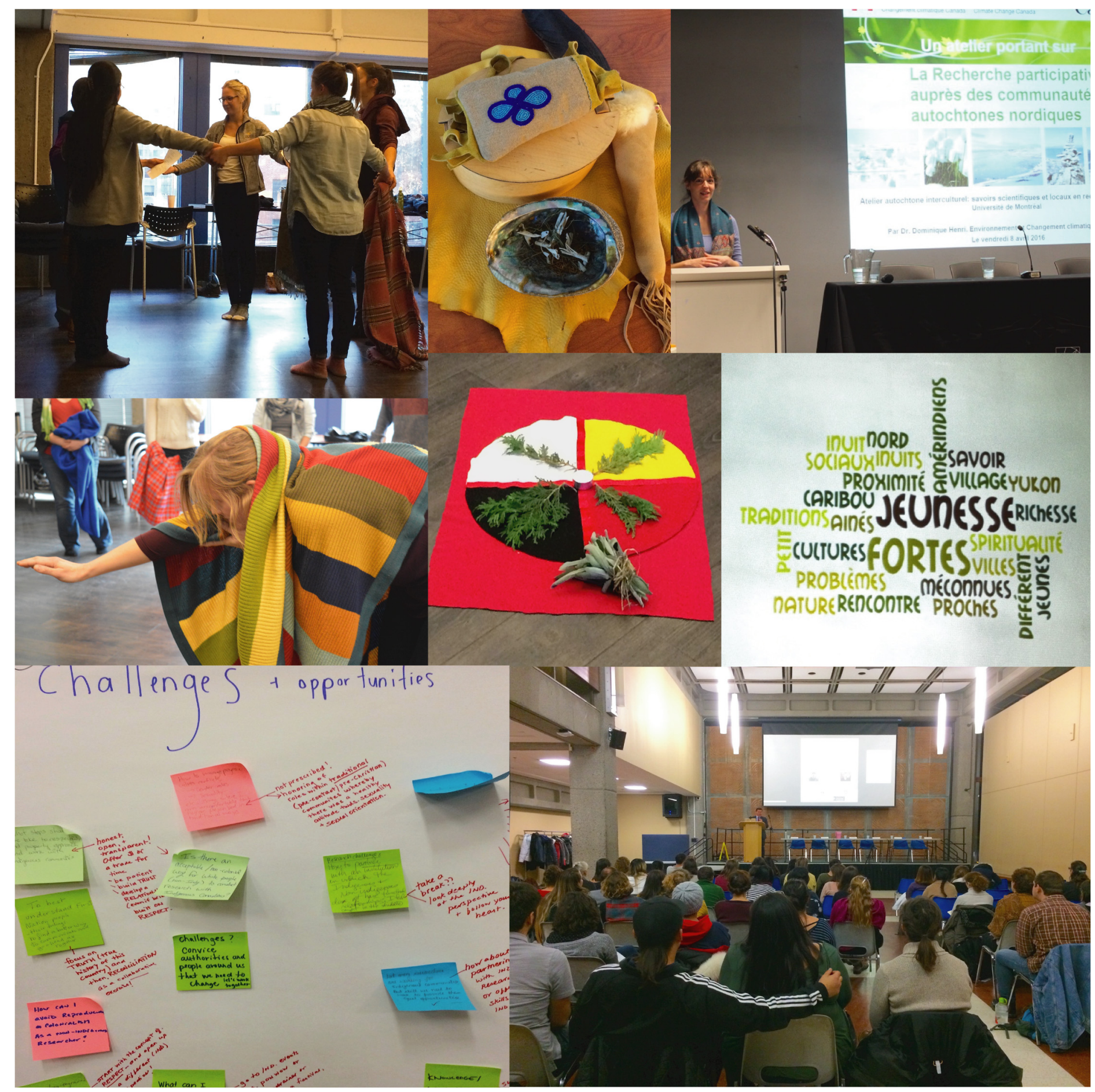

Fig. 2. Photo mosaic showing the physical environments of the Intercultural Indigenous Workshops, as well as examples of the interactive activities. 
Another theme that emerged from our analysis was the need for skillful moderation at the workshops. Skillful moderation was not only needed for logistical aspects, such as staying on schedule and managing the flow of the activities, but also to ensure that participants had sufficient time and space to reflect on workshop content. Although participants may have enrolled in our workshops to receive professional training, the experiential learning activities brought some participants on a more personal journey. The in-depth and experiential learning activities that focused on the history of colonization and current realities of Indigenous peoples in Canada fostered personal reflections among participants (Castleden et al. 2013), and for some it evoked feelings of vulnerability and sadness. As one organizer from 2017 stated: "Participating in this kind of experience for the first time can be intense and traumatic; and how participant reactions are received and dealt with is extremely important." The need for sufficient time for reflection and emotional guidance (e.g., from an Indigenous Elder) was reiterated by many participants as important while learning about emotionally difficult information, such as teachings on colonial history for participants self-identifying as settlers. Skillful moderation was thus an essential component for raising awareness about Indigenous histories, cultures, and realities. Future workshops should try and ensure that time is set aside for participants to process information on difficult subjects, for example by allowing multiple breaks during which facilitators remain available to talk with participants.

Despite the challenges of navigating complex issues in a cross-cultural environment, moderation of the workshops was generally successful. Workshops organizers recognized the importance of moderation beforehand and provided participants with space (e.g., breakout discussions) and emotional guidance (e.g., Indigenous Elders and experienced Indigenous facilitators) to encourage reflection. Feedback from attendees suggested that many were able to find space to reflect upon their experiences in a safe environment. Peer-led moderation may have contributed to fostering a safe environment that promoted communication and cooperative learning among participants (Johnson et al. 1998; Preszler 2009). In fact, the nonhierarchical structure of a peer-led workshop, which promotes knowledge sharing and includes activities with facilitators or guides as opposed to teachers or experts, may have allowed for safer sharing and a deeper connection among participants (Castleden et al. 2013). One participant reported: "I felt that I was able to listen and hear what other people were doing. It was great to hear about people's struggles, but that they were seeking out like-minded people and eager to help find solutions." Achieving a safe and inclusive environment where participants felt comfortable sharing perspectives was integral to the positive learning experience of participants and facilitators at these workshops.

\section{Workshop outcomes}

A nice opportunity for students to improve their knowledge of Indigenous contexts, to exchange, and to share their concerns.

Workshop facilitator in 2016

Our evaluation showed that the workshops were successful in cultivating cultural awareness among participants, as well as in promoting the sharing of Indigenous and non-Indigenous ways of knowing. The four themes discussed in previous sections (scope and interdisciplinarity, Indigenous representation, workshop environment, and skillful moderation) were instrumental to achieving workshop objectives. Overall, the interdisciplinary and participatory nature of the peer-led Indigenous Intercultural Workshops and the presence of Indigenous representatives provided a safe environment where participants could exchange and reflect on important research issues in Canada. We suggest that this type of training for ECRs can influence approaches to research, as represented in Fig. 3; it illustrates the circular relationship between researcher training (on the bottom half) and the potential impacts of training on research practices with Indigenous communities (on the top half). 


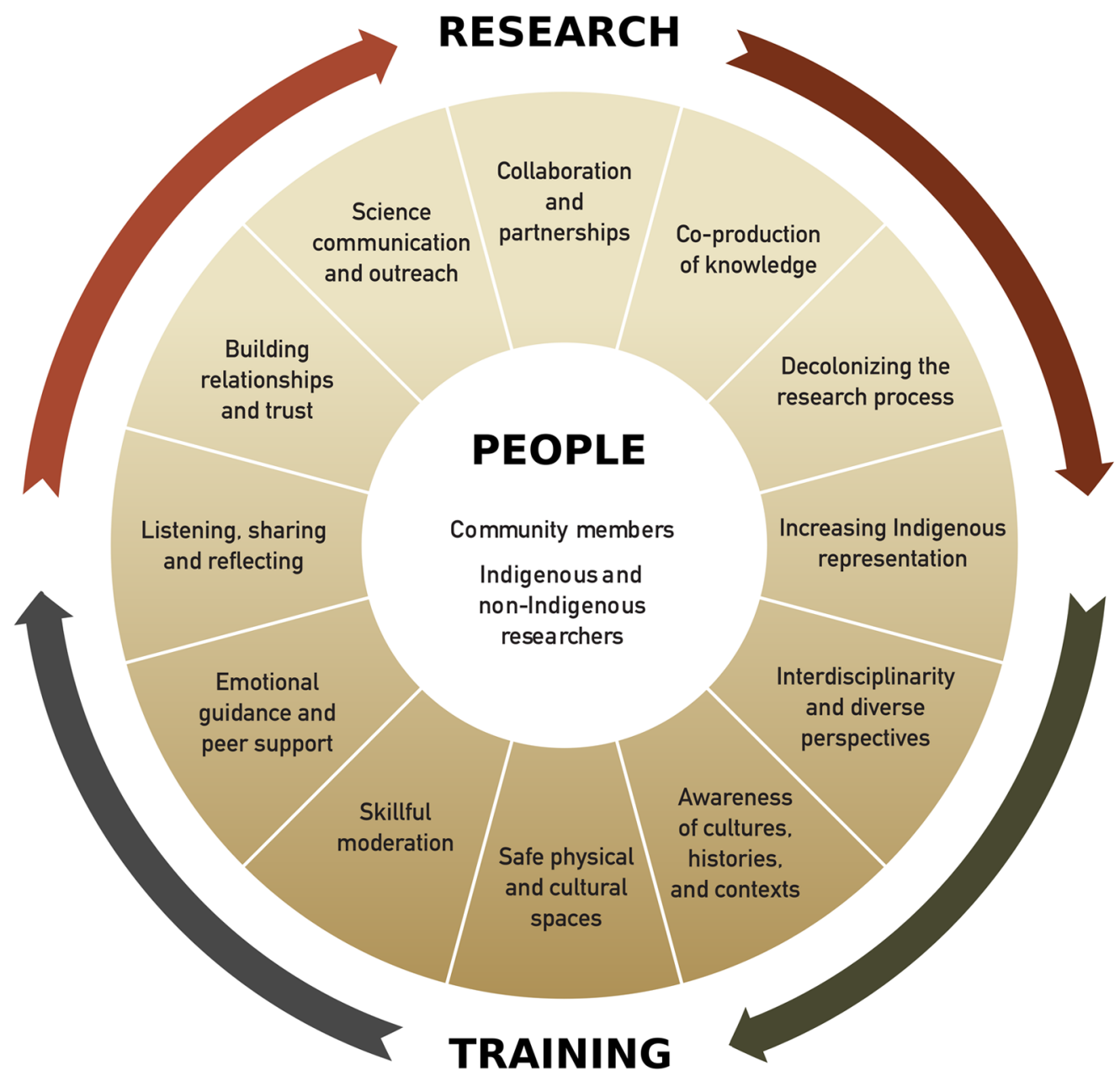

\section{"Just organizing something like this is a powerful thing. \\ Indigenous peoples are often forgotten in research and it's events like these that move us closer to better relationships" \\ - Workshop facilitator in 2017 -}

Fig. 3. This diagram shows an iterative approach to training and research that can help move us towards a new research paradigm. The cycle starts with increasing Indigenous representation (on the right side) and moves from right to left, with our understanding of collaborative research deepening at each turn. People are found at the center of this process. The training section (bottom half) shows the key themes from our evaluation of the Intercultural Indigenous Workshops and the top half shows the larger context of research shifting toward collaborative approaches.

People are at the heart of this learning process where our understanding deepens with every turn, and where community members and researchers work together towards shifting the research paradigm.

In addition, the workshop organizers-all ECRs-learned how to contribute to improving training for their peers through hosting workshops. Skills developed by organizers during the workshops ranged from identifying and inviting facilitators to coordinating the logistics (e.g., writing funding proposals, budgeting, travel expenses) and moderating activities with challenging content on colonial history. Workshop organizers learned by doing, by seeking advice, and by soliciting recommendations for 
future events. Many of the workshop organizers were asked to help organize similarly themed workshops after the events. This highlights the need for peer-led training and that the knowledge and skills developed by the organizers may help other groups improve training for ECRs. In-depth discussions between attendees have also continued since the workshops and suggest that these activities sparked important conversations about community-collaborative research and ECR training.

This paper highlights the potential of the Intercultural Indigenous Workshops to increase cultural awareness and ECRs' capacity to conduct collaborative research. It was difficult, however, to evaluate if the workshops contributed to changing how participants actually conduct their research. To evaluate this, the organizers would have had to assess if participants' research evolved toward increased integration of community-collaborative approaches as a direct result of the workshops (Cervero 1984). This type of analysis was beyond the scope of this paper, but could contribute greatly to our understanding of the impact of peer-led training on ECRs' approaches to collaborative research. Nonetheless, the fact that these workshops drew broad participation and high general appreciation may be indicative of a shift in mainstream approaches to research among ECRs.

\section{Conclusion}

Efficient? No. One workshop cannot be enough. You would need many more workshops, spread over a year or at least a semester.

Workshop facilitator in 2016

Although peer-led learning is not a substitute for formal teaching methods, it can be an effective tool to help ECRs develop "reflective practice and critical self-awareness" while being a pragmatic response to limited resources at universities (Boud 2001, p. 11). We propose that peer-led workshops integrating Indigenous representation, interdisciplinary discussions, safe physical and cultural spaces, skillful moderation, and emotional guidance can be useful to ECRs who work with Indigenous communities. While peer-led workshops cannot replace the invaluable experience gained through working directly with Indigenous communities, they can be extremely helpful to ECRs who are starting their degrees and who have little or no hands-on experience in communities. Peer-led workshops may also be useful to more experienced ECRs by building a community of practice where ECRs can share their experiences and learn through interacting with their peers. This type of training provides ECRs with a place to reflect on how research is conducted in Canada, supporting Indigenous calls for inclusive research practices and, ultimately, for the decolonization of research (Fig. 3).

One important question remains after our evaluation of these workshops: can raising cultural awareness, promoting the sharing of diverse perspectives, and teaching collaborative research approaches lead to measurable change in research practices? As expressed in the above quote, attending a 1- or 2-d event may not be sufficient to meaningfully change the way ECRs conduct their research. It is likely that regardless of intentions, ECRs will still struggle to implement behavioural changes when faced with academic demands that make engagement with Indigenous communities challenging (Beagan 2003). Peer-led workshops are therefore an important but insufficient step towards more collaborative research practices in Canada. Having ongoing, accessible, and academically recognized training for all researchers that brings Indigenous and non-Indigenous participants together can help us challenge current perspectives and make fundamental changes to how we conduct research in Canada. Although it may be unsettling, ECRs and other researchers must let down their guard and learn from different ways of knowing-for the betterment of research processes in Canada.

\section{Acknowledgements}

We acknowledge that the land on which we hosted the two Intercultural Indigenous Workshops is the traditional and unceded territory of the Kanien'keha:ka (Mohawk), a place that has long served as a 
site of meeting and exchange amongst nations. We are grateful for the participation of all workshop facilitators and participants. Heartfelt thanks to our workshop co-organizers: Geneviève Dubois, Élise Morin, Cécile de Sérigny, and Dana Simon. Thanks to Ariane Petitclerc for her graphic design work. Thank you to David Loutfi for his assistance with qualitative data analysis and proofreading. Thanks to Beatriz Osorio for assistance for the organization of the 2017 workshop and to the Fédération des Associations Étudiantes du Campus de l'Université de Montréal, Projets d'Initiatives Étudiantes (Université de Montréal), CREATE Mine of Knowledge (National Sciences and Engineering Research Council, NSERC), and McGill North for providing workshop funding. Thank you to the Quebec Centre for Biodiversity Science, Canada's National Council of the Association of Polar Early Career Scientists, and the Association of Polar Early Career Scientists for providing website support, a webinar platform, and for helping to promote the events. Many thanks to Kitikmeot Foods, Kwe Kwe Gourmet, and Chef Swaneige Traiteur for providing food and catering during workshops. Scholarships and grants were provided to the authors by NSERC (Alexander Graham Bell, Vanier), Fonds de recherche du Québec - Nature et technologies, the W. Garfield Weston Foundation, Richard J. Schmeelk Canada, Canada First Research Excellence Fund Sentinel North, CREATE Environmental Innovation (NSERC), the Northern Scientific Training Program, and the Marine Environmental Observation Prediction and Response network. Our thanks to three anonymous reviewers whose constructive comments helped improve this manuscript.

\section{Author contributions}

GAM, MF, and CG conceived and designed the study. GAM, MF, CG, and JL-B collected the data. GAM, MF, CG, SD-B, JL-B, AKM, and DAH analyzed and interpreted the data. JL-B contributed resources. GAM, MF, CG, SD-B, JL-B, AKM, and DAH drafted or revised the manuscript.

\section{Competing interests}

The authors have declared that no competing interests exist.

\section{Data availability statement}

All relevant data are within the paper and in the Supplementary Material.

\section{Supplementary material}

The following Supplementary Material is available with the article through the journal website at doi:10.1139/facets-2018-0046.

Supplementary Material 1

\section{References}

Adams MS, Carpenter J, Housty JA, Neasloss D, Paquet PC, Service C, et al. 2014. Toward increased engagement between academic and indigenous community partners in ecological research. Ecology and Society, 19(3): 5. DOI: 10.5751/ES-06569-190305

Association of Canadian Universities for Northern Studies (ACUNS). 1982. Ethical principles for the conduct of research in the North. ACUNS, Ottawa, Ontario. 9 p.

Ayre ML, Wallis PJ, and Daniell KA. 2018. Learning from collaborative research on sustainably managing fresh water: implications for ethical research-practice engagement. Ecology and Society, 23(1): 6. DOI: 10.5751/ES-09822-230106 
Beagan BL. 2003. Teaching social and cultural awareness to medical students: "it's all very nice to talk about it in theory, but ultimately it makes no difference." Academic Medicine, 78: 605-614. PMID: 12805041 DOI: 10.1097/00001888-200306000-00011

Boud D. 2001. Making the move to peer learning. In Peer learning in higher education: learning from and with each other. Edited by D Boud, R Cohen, and J Sampson. Kogan Page, London, UK. pp. 1-21.

Bousquet M-P. 2012. De la pensée holistique à l'Indian Time : dix stéréotypes à éviter sur les Amérindiens. Nouvelles pratiques sociales, 24: 204-226. DOI: 10.7202/1016356ar

Bridle H, Vrieling A, Cardillo M, Araya Y, and Hinojosa L. 2013. Preparing for an interdisciplinary future: a perspective from early-career researchers. Futures, 53: 22-32. DOI: 10.1016/ j.futures.2013.09.003

Brunet ND, Hickey GM, and Humphries MM. 2014a. The evolution of local participation and the mode of knowledge production in Arctic research. Ecology and Society, 19(2): 69. DOI: 10.5751/ES06641-190269

Brunet ND, Hickey GM, and Humphries MM. 2014b. Understanding community-researcher partnerships in the natural sciences: a case study from the Arctic. Journal of Rural Studies, 36: 247-261. DOI: 10.1016/j.jrurstud.2014.09.001

Canadian Institutes of Health Research, Natural Sciences and Engineering Research Council of Canada, and Social Sciences and Humanities Research Council of Canada. 2014. Tri-council policy statement: ethical conduct for research involving humans. Secretariat on Responsible Conduct of Research, Ottawa, Ontario, Canada, 210 p.

Castleden H, Morgan VS, and Lamb C. 2012. "I spent the first year drinking tea": exploring Canadian university researchers' perspectives on community-based participatory research involving Indigenous peoples. The Canadian Geographer/Le Géographe canadien, 56: 160-179. DOI: 10.1111/j.15410064.2012.00432.x

Castleden H, Daley K, Morgan VS, and Sylvestre P. 2013. Settlers unsettled: using field schools and digital stories to transform geographies of ignorance about Indigenous peoples in Canada. Journal of Geography in Higher Education, 37: 487-499. DOI: 10.1080/03098265.2013. 796352

Cervero RM. 1984. Evaluating workshop implementation and outcomes. New Directions for Adult \& Continuing Education, 1984: 55-67. DOI: 10.1002/ace.36719842207

Deakin H, Wakefield K, and Gregorius S. 2012. An exploration of peer-to-peer teaching and learning at postgraduate level: the experience of two student-led NVivo workshops. Journal of Geography in Higher Education, 36: 603-612. DOI: 10.1080/03098265.2012.692074

First Nations Information Governance Centre. 2014. Ownership, control, access and possession (OCAP): the path to First Nations information governance. The First Nations Information Governance Centre, Ottawa, Ontario. 49 p.

Ford JD, Knight M, and Pearce T. 2013. Assessing the 'usability' of climate change research for decision-making: a case study of the Canadian International Polar Year. Global Environmental Change, 23: 1317-1326. DOI: 10.1016/j.gloenvcha.2013.06.001 
Gagnon CA, and Berteaux D. 2009. Integrating traditional ecological knowledge and ecological science: a question of scale. Ecology and Society, 14(2): 19 [online]: Available from ecologyandsociety.org/vol14/iss2/art19/. DOI: 10.5751/ES-02923-140219

Government of Canada (GC). 2014. Panel on research ethics: research involving the First Nations, Inuit and Métis peoples of Canada. Chapter 9. In Tri-council policy statement: ethical conduct for research involving humans. Canadian Institutes of Health Research, Natural Sciences and Engineering Research Council of Canada, and Social Sciences and Humanities Research Council of Canada. Secretariat on Responsible Conduct of Research, Ottawa, Ontario, Canada, pp. 105-133 [online]: Available from pre.ethics.gc.ca/eng/policy-politique/initiatives/tcps2-eptc2/ chapter9-chapitre $9 /$.

Hall BL. 2005. In from the cold? Reflections on participatory research from 1970-2005. Convergence, 38: 5-24.

Institut Nordique du Québec (INQ). 2017. INQ: research guidelines: First Peoples Working Group. Groupe de travail des Premiers Peuples de l'Institut Nordique du Québec, INQ, Québec City, Québec. $16 \mathrm{p}$.

Inuit Tapiriit Kanatami (ITK). 2018. National Inuit strategy on research. ITK, Ottawa, Ontario, Canada. $44 \mathrm{p}$.

Jack SM, Brooks S, Furgal CM, and Dobbins M. 2010. Knowledge transfer and exchange processes for environmental health issues in Canadian Aboriginal communities. International Journal of Environmental Research and Public Health, 7: 651-674. PMID: 20616996 DOI: 10.3390/ ijerph7020651

Johnson DW, Johnson RT, and Smith KA. 1998. Cooperative learning returns to college what evidence is there that it works? Change: The Magazine of Higher Learning, 30(4): 26-35. DOI: $10.1080 / 00091389809602629$

Johnson N, Alessa L, Behe C, Danielsen F, Gearheard S, Gofman-Wallingford V, et al. 2015. The contributions of community-based monitoring and traditional knowledge to Arctic observing networks: reflections on the state of the field. Arctic, 68(5): 28-40. DOI: 10.14430/arctic4447

Kitchen J, and Raynor M. 2013. Indigenizing teacher education: an action research project. The Canadian Journal of Action Research, 14: 40-58.

Koster R, Baccar K, and Lemelin RH. 2012. Moving from research ON, to research WITH and FOR Indigenous communities: a critical reflection on community-based participatory research. The Canadian Geographer/Le Géographe canadien, 56: 195-210. DOI: 10.1111/j.1541-0064.2012.00428.x

Kovach M. 2009. Indigenous methodologies: characteristics, conversations, and contexts. University of Toronto Press, Toronto, Ontario. 216 p.

Larivière V, Ni C, Gingras Y, Cronin B, and Sugimoto CR. 2013. Bibliometrics: global gender disparities in science. Nature, 504(7479): 211-213. PMID: 24350369 DOI: 10.1038/504211a

Mantyka-Pringle CS, Jardine TD, Bradford L, Bharadwaj L, Kythreotis AP, Fresque-Baxter J, et al. 2017. Bridging science and traditional knowledge to assess cumulative impacts of stressors on ecosystem health. Environment International, 102: 125-137. PMID: 28249740 DOI: $10.1016 /$ j.envint.2017.02.008 
Mercier OR, and Leonard B. 2000. Indigenous knowledge(s) and the sciences in global contexts: bringing worlds together. University of Toronto Press, Toronto, Ontario. $432 \mathrm{p}$.

Mitchell TL, and Baker E. 2005. Community-building versus career-building research: the challenges, risks, and responsibilities of conducting research with Aboriginal and Native American communities. Journal of Cancer Education, 20: 41-46. PMID: 15916520 DOI: 10.1207/s15430154jce2001s_10

Nunavut Research Institute (NRI) and Inuit Tapiriit Kanatami (ITK). 2007. Negotiating research relationships with Inuit communities: a guide for researchers. Edited by S Nickels, J Shirley, and G Laidler. Inuit Tapiriit Kanatami and Nunavut Research Institute, Ottawa, Ontario and Iqaluit, Nunavut. 38 p.

Ouellet Dallaire C, Trincsi K, Ward MK, Harris LI, Jarvis L, Dryden RL, et al. 2018. Creating space for sustainability literacy: the case of student-centered symposia. International Journal of Sustainability in Higher Education, 19: 839-855. DOI: 10.1108/IJSHE-08-2017-0126

Preszler RW. 2009. Replacing lecture with peer-led workshops improves student learning. CBE-Life Sciences Education, 8: 182-192. DOI: 10.1187/cbe.09-01-0002

Sandelowski M. 2000. Whatever happened to qualitative description? Research in Nursing \& Health, 23: 334-340. PMID: 10940958 DOI: 10.1002/1098-240X(200008)23:4<334::AID-NUR9>3.0.CO;2-G

Sjöberg Y, Gomach S, Kwiatkowski E, and Mansoz M. 2019. Involvement of local Indigenous peoples in Arctic research-expectations, needs and challenges perceived by early career researchers. Arctic Science, 5(1): 27-53. DOI: 10.1139/as-2017-0045

Tobias JK, Richmond CAM, and Luginaah I. 2013. Community-based participatory research (CBPR) with Indigenous communities: producing respectful and reciprocal research. Journal of Empirical Research on Human Research Ethics, 8: 129-140. PMID: 23651937 DOI: 10.1525/jer.2013.8.2.129

Tondu JME, Balasubramaniam AM, Chavarie L, Gantner N, Knopp JA, Provencher JF, et al. 2014. Working with northern communities to build collaborative research partnerships: perspectives from early career researchers. Arctic, 67: 419-429.

Vogel L. 2015. The new ethics of Aboriginal health research. Canadian Medical Association Journal, 187(5): 316-317. PMID: 25691784 DOI: 10.1503/cmaj.109-4998

Wilson S. 2008. Research is ceremony: Indigenous research methods. Fernwood Publishing, Black Point, Nova Scotia. 144 p. 